\title{
La realidad absoluta de las categorías
}

\begin{abstract}
El problema de las categorías ha sido objeto de debate desde que Aristóteles formulara su órganon o método de estudio de las cosas. Tradicionalmente las categorías han suscitado el problema de su naturaleza, con distintas soluciones; su número (hay quienes defienden que su número es indeterminado y quienes dicen que son sólo diez); su simetría (¿están hechas todas con el mismo criterio?); y como elementos que responden a un determinado modo de conocer. En este artículo nos planteamos el problema de la realidad de las categorías, distinguiendo muchos aspectos, uno de los cuales es la realidad absoluta de las mismas. De esta manera hacemos una hermenéutica, la hermenéutica de las categorías.
\end{abstract}

\section{Palabras clave}

categorías, determinación lingüística, nivel universal de la determinación lingüística, realidad, cosas, abstracto, creación cognoscitiva.

\begin{abstract}
The debate about categories has been in the Western Civilization since Aristotle formulated his organon or set of procedures to study things. In the European tradition categories have prompted different problems: the problem of their nature, with different solutions; the problem of their number (some say it is indefinite and some it is restricted to ten); the criterium in accordance with which they have been created; and finally as the result of the different modes of knowing. In this article the problem of reality of categories is to be posed, separating in it many aspects, one of which constitute the absolute reality of categories. In this sense our study is hermeneutics, the hermeneutics of categories.
\end{abstract}

\section{Keywords}

categories, linguistic determination, universal level of linguistic determination, reality, things, abstract, cognizant creation.

\section{La realidad de las categorías.}

El problema de la realidad de las categorías tiene que ver con la función que las categorías desempeñan en un universo de conocimiento dado. Esto significa que determinar la realidad de las categorías en sí misma es una hermenéutica. La realidad de las categorías depende del

* Profesor de lingüística inglesa de la Universidad de Almería. Seguidor de Coseriu y de Ortega y Gasset, se dedica al estudio de las relaciones lenguaje-pensamiento partiendo del ser humano como ser que habla, dice y conoce, consecuencia de su doble condición de ser libre e histórico. Sus trabajos están en íntima conexión con la filosofía del lenguaje, la lingüística fundamental y la filosofía de la ciencia. Entre sus obras destacan, /An Open Dimension of Meaning/, 1997; /La intelección, el significado, los adjetivos/, 1999; /Benjamin Lee Whorf y el problema de la intelección/, 2001; /Significado y conocimiento,/ 2002; /La lingüística del decir, el logos semántico y el logos apofántico,/ 2004; /Los fundamentos de la teoría de Chomsky. Revisión crítica/, 2006; /Facts of Speech and Facts of Evolution,/ 2006; /La lingüística cognitiva, análisis y revisión,/ 2008; /Las relaciones lenguaje-pensamiento o el problema del logos/, 2010; /Sobre las categorías,/ 2011. Correo electrónico,jesus.gerardo@ual.es 
punto de vista desde el que se consideren. Según la función que desempeñan, de antemano podemos ver las categorías:

A. como elementos que van más allá de las lenguas siendo en sí mismos la manifestación del pensamiento, -nivel universal de la determinación lingüística. Determinada así la realidad absoluta o universal de las categorías, podremos preguntarnos

i. si las categorías son categorías de la mente o categorías de las cosas;

ii. si las categorías se forman por lo que las cosas tienen en común, por la experiencia o por analogía;

iii. y si, con Aristóteles, necesitamos de algún tipo de conocimiento especial para concebir las categorías;

B. Podremos también concebir las categorías como elementos que pertenecen a una lengua, -nivel histórico de la determinación lingüística;

C. como elementos que son la manifestación del modo de pensar de su autor -nivel individual de la determinación lingüística;

D. como elementos que constituyen un decir;

E. nos podremos preguntar igualmente por la finalidad según la cual las categorías son concebidas;

F. y, por último, nos podremos preguntar por la cohesión del criterio según el cual las categorías fueron concebidas.

A continuación vamos a estudiar el problema de la realidad absoluta de las categorías, es decir, vamos a estudiar las categorías en el nivel universal de la determinación lingüística, nivel que tiene que ver con el hablar en sí y con las condiciones del hablar.

\section{La realidad absoluta o universal de las categorías.}

2.1. El problema de las categorías como realidad absoluta, puede formularse así: ¿Cuál es la razón última de la existencia de las categorías? ¿Qué base real o cognoscitiva tienen las categorías? Se trata, como ya hemos dicho, del estudio de las categorías en el nivel universal de la determinación lingüística. En consecuencia, ya no hablamos de las categorías de Aristóteles o de cualquier otro sistema de categorías, sino de las categorías en cuanto que son tales y desempeñan una función, es decir, hablamos de la esencia de las categorías.

2.2.1. La argumentación de Aristóteles sobre las categorías gira en torno al concepto de lo que es la substancia. Como él mismo nos dice, "toda substancia parece significar un "esto",1, es decir, toda substancia siendo la pura condición de ser es, además, un algo, asimilándose a lo que son las cosas, que se pueden tocar: un algo que es en sí como cosa objetiva. De esta manera la substancia es y, en virtud de que es, se objetiva como algo que es en sí, con lo que su condición de ser creada al decir se asimila a lo que es de manera física y concreta.

2.2.2. La substancia es la primera de las categorías y lo dicho se aplica de ella de manera privativa. Pero Aristóteles también trata de esta manera a las otras categorías (el cuánto, el cuál, el relativamente a qué, el dónde, el cuándo, el estar en postura, el estar en un estado, el hacer y el padecer): son realidades objetivas que participan de la pura condición de ser siendo en otra cosa, en la que encontraremos la realidad y ser de la cosa y la substancia que

$1 \quad$ Categorías $3 \mathrm{~b} 10$. 
Aristóteles atribuye a todo lo que es. De esta manera la substancia es cosa objetiva y las otras categorías son también cosas objetivas, con la particularidad de que son en otra cosa.

2.3. Una vez que Aristóteles deja bien sentado que la substancia es, busca relaciones que se dan, no en la substancia, sino en las cosas a las que se aplica la substancia, relaciones que en realidad, si bien parten de un concepto creado por analogía que conviene a las cosas materiales, no son más que consideraciones cognoscitivas que se añaden al compuesto de cosa objetiva más condición pura de ser (=substancia). El supuesto de que todo lo que es es substancia constituye la base para poder hablar de las categorías. El darse en otra cosa, aparte del darse en sí, constituye una nueva forma de ser que Aristóteles pone de manifiesto en las Categorías. Lo particular de la existencia de las categorías es que Aristóteles las concibe justamente como dándose en muchas cosas y en virtud de que se dan en esas muchas cosas Aristóteles las concibe como realidades objetivas de las cuales se puede decir o predicar. Pero constituyen en sí mismas una abstracción, por consiguiente, un objeto mental.

2.4. Aristóteles no se ha planteado nunca si las cosas reales existen, con lo cual tampoco tiene en cuenta la labor del sujeto cognoscente. Tampoco se pregunta por el grado de realidad de aquello de que habla en las Categorías. Da por supuesto que las cosas con las que se encuentra son y, puesto que son, busca el fundamento de su ser en lo que él intuye como substancia. Ahora bien, si la substancia es un algo que Aristóteles intuye en las cosas y que va a buscarlo en las mismas, las otras categorías (el cuánto, el cuál, el respecto de, etc.), que no se pueden concebir sino en otra cosa, que a la vez constituye también una substancia, serán así mismo algo concebido como intuición del propio Aristóteles, es decir, serán algo que Aristóteles busca en las cosas o seres, a pesar de que las ha creado sobre la base de las cosas por intuición y analogía. Luego, tanto la substancia como las otras categorías tendrán el mismo grado de realidad: responden a lo que es el acto del conocer, el acto de aprehender la realidad mentalmente a partir de una sensación inicial o intuición primera o, como él mismo dice, una aísthesis ${ }^{2}$. Las categorías en su realidad más radical son instrumentos a priori del conocer humano porque se dan y existen en el acto del conocer y gracias a las cuales, en sentido contrario, existe el propio conocer humano. Las categorías así son absolutas y se dan necesariamente. Sin categorías, sean del tipo que sea, no hay conocimiento propiamente humano. Puede haber, sí, una intuición o aísthesis, que se podrá desarrollar según su propia naturaleza, es decir, sensiblemente. La aísthesis, que es sensación, algo sensible y concreto, constituye también conocimiento. Y ésta es la línea de demarcación que separa lo que es el conocimiento sensible, conocimiento que tienen tanto los humanos como los animales, el moverse por impulsos sensibles, y el conocimiento propiamente humano, conocimiento creativo fruto de la libertad humana, que transforma lo sensible y concreto en abstracto, lo abstracto en idea y la idea en contenido de conciencia ${ }^{3}$. El conocimiento humano, que necesariamente parte de lo sensible y concreto, de una intuición o aísthesis, transforma lo que llega al sujeto a través de sus sentidos y lo hace otra cosa de lo que es. Este hacer otra cosa de lo sensible y concreto es la pura creación y la pura abstracción (=transformación de la forma de ser de aquello que aprehendemos ${ }^{4}$ ), lo cual nos da el elemento manipulable y acomodaticio

\footnotetext{
De Anima III, 1, 425a14 apud Ortega y Gasset 1992: 128.

Cf. Martínez del Castillo 1999: cap. 3.

Recordemos que Ortega y Gasset llama a la abstracción "extracción” (cf. 1992: 64). Cuando abstraemos extraemos una cosa que está entre muchas otras, dejándola aislada (cf. Ortega y Gasset 1996: 107), con lo que esto que es abstraído no es en sí mismo sino que es considerado como si fuera. Luego lo transformamos desde lo que es en algo que no es.
} 
que distingue al conocer humano. Y este elemento manipulable y acomodaticio es lo que constituye el ser o la substancia o la categoría ${ }^{5}$. Lo abstraído, pura invención, es la categoría, instrumento a priori del conocimiento, es decir, instrumento sin el cual no se daría el conocimiento humano, ni la substancia, ni el ser.

2.5. Ahora bien, la realización de la categoría es ejecutada siempre por un sujeto individual creativo e inteligente incardinado en una circunstancia. Si bien el ser humano, allá donde se encuentre, conoce, es decir, transforma lo sensible en abstracto hasta llegar a constituir la categoría o clase de cosas que aplica libremente a lo que aprehende de forma individual, necesita de lo material para comunicarse con otro ${ }^{6}$. Para que una conciencia humana se pueda comunicar con otra conciencia necesita medios concretos y materiales. Lo necesario no se da en sí mismo. Dada la limitación del sujeto creador las categorías, en sí mismas necesarias y a priori, se tienen que realizar en lo contingente, es decir, en formas de validez limitada. Dada también la condición histórica del sujeto, las formas tienen que ser participadas y comunes dentro de una comunidad de hablantes. La realización de las categorías siempre se da en una lengua. De esta manera, la categoría se materializa en formas concretas individuales que se han formado sobre la base de formas compartidas y comunes en una comunidad lingüística, es decir, formas históricas. Si bien en sí mismas las categorías son necesarias, en su realización siempre son algo contingente, e incluso, algo fugaz. La existencia necesaria de las categorías es cosa distinta de su realización.

2.6. El acto del conocer, que empieza con la aísthesis, es un acto de transformación en el que el sujeto fabrica, es decir, inventa constructos extraídos de la aísthesis que sólo tiene él como individuo, los modifica, les da un grado de realidad determinado, les atribuye una universalidad infinita de designación, los relaciona, les da un nombre, los determina para que se apliquen de las cosas reales, y los convierte en palabras y constructos de una lengua que pueden ser tanto individuales como comunes o ambas cosas a la vez ${ }^{7}$. Con esto el acto del conocer crea las categorías y éstas hacen posible el acto del conocer. El acto del conocer es un acto de creación individual, por un lado, y un acto absoluto, por otro, que pasa de lo necesario a lo contingente, de lo universal a lo histórico e individual, siendo de esta manera un acto de creación histórica ${ }^{8}$. El acto del conocer es un acto de traducción del lenguaje de los sentidos al lenguaje del ser -ese algo creado en virtud de la aplicación de la categoría al constructo inicialmente sensible y ahora transformado. La aísthesis, en virtud del acto del conocer, de ser concreta y sensible y como tal efímera, se hace otra cosa distinta de lo que es ${ }^{9}$. De esta

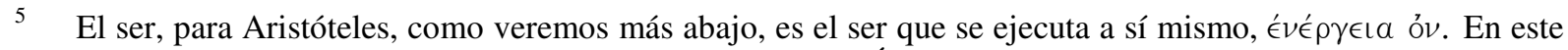
sentido la existencia del ser se identifica con su esencia. Ésta no es más que lo que representa la categoría. El caballo es "el ser que caballea”. El ser del caballo está en la categoría que lo representa.

6 Coseriu distingue dos clases de comunicación: comunicar algo a alguien, cuyo contrario, el no comunicar, no llega a negar la naturaleza lingüística del hablar, y el comunicar con alguien, condición imprescindible del hablar o lenguaje. El comunicar con alguien constituye la segunda dimensión del lenguaje, la historicidad, que se basa en la alteridad del lenguaje, la cual se corresponde con la alteridad del ser humano. La dimensión de la alteridad -condición esencial del ser humano: "ese 'ser-con-otro'- el reconocerse a sí mismo en otros, el reconocer en el 'tú' a otro 'yo' [...], coincide con la intersubjetividad originaria de la conciencia: con el hecho de que la conciencia humana es conciencia abierta hacia otras conciencias con las que establece comunicación, es decir, les reconoce las mismas facultades de sentir, pensar, juzgar, significar e interpretar. Y el lenguaje es la forma básica de este salir la conciencia de sí misma e ir al encuentro de otras conciencias" (cf. Coseriu 2006: 44).

7 Cf. Martínez del Castillo 2004.

8 Este acto de creación histórica podrá constituir un hecho de lengua o un hecho de discurso.

9 Cf. Ortega y Gasset 1992; cf. Martínez del Castillo 2004. 
manera el lenguaje, mediador necesario en el conocer humano, se convierte en la posibilidad del ser. Dice Ortega y Gasset:

pertenece a la realidad que el hombre esté ante ella y la vea. Casi son equivalentes los términos perspectiva y conocimiento. [...] El conocimiento no es sólo un modus cognoscentis sino una positiva modificación de lo conocido. [...] El conocimiento [...] es perspectiva, por tanto, ni propiamente un ingreso de la cosa en la mente [...], ni un estar la "cosa misma" en la mente per modum cognoscentis [...] ni es una copia de la cosa [...], ni una construcción de la cosa [...], sino una "interpretación" de la cosa misma sometiéndola a una traducción [...] del lenguaje del ser, que es mudo, al lenguaje decidor del conocer. Este lenguaje al que es traducido es, ni más ni menos, el lenguaje, el lógos. Conocer en su

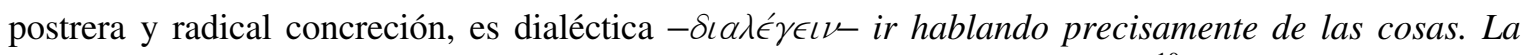
palabra enuncia las vistas en que nos son patentes los aspectos de la Realidad ${ }^{10}$.

2.7. Las categorías son, pues, instrumentos a priori del conocer, por un lado, instrumentos contingentes del conocer, es decir, históricos, por otro, e incluso, instrumentos individuales de conocer, por consiguiente, efímeros. Estos tres aspectos se dan siempre juntos y en el mismo acto del conocer. Ahora bien, todo acto de conocer humano es un acto complejo que es también un decir y un hablar. En este sentido, las categorías son siempre categorías lingüísticas: las categorías son hechas según los parámetros de la lengua y son, por tanto, formas comunes y participadas o formas individuales hechas sobre bases comunes y participadas, categorías que, por encima de su individualidad concreta, están vigentes de una forma u otra en una comunidad de hablantes y que como tal no existen más que virtualmente en la forma de ver y concebir la realidad de los hablantes de una lengua.

2.8.1. Los cognitivistas interpretan las categorías únicamente como universales, es decir, como cosas que se dan siempre independientemente de su valor como elementos desgajados de una lengua y creados por un sujeto individual. Para los cognitivistas, esta universalidad es de tipo estructural, tanto biológica, como genética o culturalmente. Es decir, los cognitivistas atribuyen dicha universalidad a causas somáticas, psicológicas, sociológicas e, incluso, genéticas $^{11}$. En consonancia, las categorías son consideradas como cosas concretas y objetivas que se dan en todos los individuos pertenecientes al género humano. La prueba que aducen para defender esta concepción es que las categorías son el resultado de la comprobación empírica. Apoyan la universalidad de las categorías en lo que llaman la categorización, un proceso que se da en el hombre ${ }^{12}$ y en la estructura de la mente, que por otro lado, defienden, está "corporeizada"

2.8.2. El recurso de los cognitivistas a la experimentación de las categorías se basa en la confusión de los conceptos de "histórico", lo que es común y participado entre los hablantes de una lengua, y "natural". Los cognitivistas, que no conocen el concepto de historicidad ni, por consiguiente, el de alteridad, asimilan lo universal a lo biológico y genético. Un hablante que nace en una historicidad dada aprende lo que está en vigor en su comunidad lingüística como único instrumento en su relacionarse con el mundo. Para el hablante 'naif' no existe

10 Ortega y Gasset 1989: 41-42.

11 Lakoff 1990: 266.

12 Dice Lakoff (1990: 8): "human categorization is essentially a matter of both human experience and imagination -of perception, motor activity, and culture, on the one hand, and of metaphor, metonymy, and mental imagery on the other. As a consequence human reason crucially depends on the same factors, and therefore cannot be characterized merely in terms of the manipulation of abstract symbols".

13 Cf. Lakoff 1990: xvi; Lakoff y Johnson 1999: 7. Para una crítica sobre la lingüística cognitiva cf. Martínez del Castillo 2008. 
más realidad que la que ha aprendido por primera y, a veces, definitiva vez. Los conocimientos así adquiridos constituyen creencias, instrumentos del conocer que se desarrollan en los individuos sin que éstos lleguen a darse cuenta. Esto es así por dos razones:

a) porque son conocimientos que se aprenden sin que los individuos que los aprenden lleguen a conocer ni plantearse el problema que llevan consigo, y

b) porque, para los individuos que así los aprenden, problema y solución, constituyen la misma cosa.

De esta forma las creencias llegan a ser consideradas como hechos incontrovertibles, hechos que son así "porque son naturales". La constatación de la existencia de las creencias como hecho que se da en los humanos se ha descrito desde muy antiguo. Ya en el siglo XIII decía Santo Tomás de Aquino:

Ea quibus a pueritia animus imbuitur, ita firmiter tenentur ac si essent naturaliter et per se nota ${ }^{14}$.

Para Ortega y Gasset, las creencias son una realidad en las que el hombre no ejerce su libertad, ya que aquéllas llegan a constituir una fe:

En la creencia, el hombre no elige su modo de creer ni de pensar, sino que, por el contrario, está desde luego sumergido en su fe, sin que sepa por dónde ha entrado en ella ni ocurrírsele querer salir de ella. $\mathrm{Su}$ creencia ni siquiera le parece creencia, sino que le parece la realidad misma ${ }^{15}$.

Humboldt por su parte describe este hecho en términos de la lengua:

Por el mismo acto por el que el hombre hila desde su interior la red de la lengua, se hace él mimo hebra de aquélla, y cada lengua traza en torno al pueblo al que pertenece un círculo del que no se puede salir si no es entrando al mismo tiempo en el círculo de otra ${ }^{16}$.

De aquí que podamos decir que el lenguaje forma el pensamiento ${ }^{17}$.

2.8.3. Ahora bien, dada la existencia de las categorías como creencias entre los hablantes de una lengua, puedo yo muy bien "experimentar", es decir, comprobar empíricamente cuáles son las categorías según las cuales este y este hablante se relaciona con el mundo. Se puede muy bien comprobar que los hablantes de una lengua utilizan las categorías de su lengua de forma espontánea y sobre todo de forma irreflexiva ${ }^{18}$. Es decir, que las categorías de una lengua constituyen un hecho que el hablante, como dice Humboldt, no puede abandonar si no es entrando en el mundo que describe, no su lengua, sino otra lengua. La comprobación

14 Summa contra gentiles I c, XI.

15 Ortega y Gasset 1989: 173

16 1990: 83. Por otro lado, dice Humboldt (1990: 32): “[el lenguaje] es indispensable [...] para acceder a una concepción del mundo a la que el hombre sólo puede llegar en la medida en que va llevando su pensamiento hacia una mayor claridad y determinación, lo que es fruto del pensar en comunidad con los demás".

17 Dice Humboldt (Humboldt 1990: 37): "El lenguaje no es un instrumento para designar objetos ya pensados, sino que es más bien el "órgano formador del pensamiento"”.

18 Los cognitivistas dirían de forma inconsciente (cf. Lakoff 1990: 6). Son dos cosas muy distintas. Decir conocimiento inconsciente es una contradicción. Dice Coseriu: "La afirmación de que un saber es inconsciente es una contradictio in adiecto. La idea de situar el saber lingüístico no en el individuo, sino en la conciencia colectiva es simplemente una solución de compromiso. Se reconoce que el saber lingüístico es un saber (bastante) seguro y que el individuo no puede justificar ese saber, pero se extraen conclusiones falsas" (Coseriu 1992: 215-16). Por otro lado, el conocimiento irreflexivo es consciente. 
empírica, pues, no conduce a nada nuevo: sólo conduce a lo que ya previamente sabíamos por razonamientos a priori o por intuición o analogía, a saber: que las creencias se dan entre los hablantes, que los hablantes identifican las categorías de su lengua con la realidad misma, y que todos los hablantes tienen sus propias creencias. Con esto se podrá concluir que las categorías son "naturales", que se dan en todos los seres humanos salvo determinados detalles propios de la lengua en cuestión, que son "inconscientes", que responden a la estructura de la mente la cual está "corporeizada", que ésta es igual para todo el género humano, y que las categorías revelan la forma como los humanos conceptualizan. ¿Es suficiente, pues, el método empírico para saber asuntos que tienen que ver con la mente y con el conocimiento humano? ¿No sabíamos de antemano y a priori ${ }^{19}$ que las categorías forman parte del conocer y que entre éstas se cuelan las creencias?

2.8.4. En consecuencia, podemos plantearnos el siguiente problema: ¿son las categorías las mismas para todos los seres humanos? Todas las categorías y todos los significados son lingüísticos. En este sentido la respuesta es no. Por tanto, para saber sobre las categorías tenemos que aplicar los mismos métodos de investigación que se aplican al lenguaje. La razón dada por Lakoff de que las categorías de la teoría clásica no eran el resultado de la investigación empírica ${ }^{20}$ no se aplica en toda su extensión. La comprobación empírica se ha de aplicar sólo al aspecto histórico de las categorías.

2.8.5. Por otro lado, Lakoff atribuye a Aristóteles lo que llama la teoría clásica de las categorías. En contra de ésta, Lakoff asegura que la interpretación de las categorías se ha de hacer conjugando la experimentación y ejerciendo la imaginación: "human categorization is essentially a matter of both human experience and imagination" ${ }^{\text {"21 }}$. Es decir que, interpretando estas palabras podemos decir que sabremos lo que son las categorías si combinamos la inducción (=método empírico) y la analogía, método, por otro lado, que usa Aristóteles cuando define sus principios y hace sus definiciones ${ }^{22}$.

2.8.6.1. Para los cognitivistas, la prueba suprema de la efectividad de la comprobación empírica y de la existencia natural de las categorías nos la da Lakoff en el hecho que toma de la lengua dyirbal, lengua aborigen australiana. Hablando de la categorización, Lakoff resalta el funcionamiento de la categoría balan del dyirbal como forma genuina y prototípica de categorizar de los humanos. Citando a R. M. W. Dixon, Lakoff dice que la lengua dyirbal hace una "excelente clasificación de las cosas". Cada vez que un hablante dyirbal utiliza una palabra que designe una cosa del mundo en una oración tiene que marcar de alguna forma la clase a la que pertenece ${ }^{23}$. De esta manera hay cuatro clases: bayi, balan, balam y bala. Pues bien, de la clase segunda, balan, forman parte las hembras, el fuego, el agua, y la lucha, interpretando Lakoff a los primeros objetos semánticos como "las mujeres" y a los tres últimos como "cosas peligrosas". De esta categoría, Lakoff toma el título de su libro: Women, Fire and Dangerous Things, uno de los libros fundacionales de la lingüística cognitiva. Y concluye Lakoff:

No obstante, el saber a priori que las categorías se dan necesariamente, no es óbice para que efectivamente comprobemos cuáles son las creencias de la "gente". Esta labor sí que es productiva por parte de la lingüística cognitiva.

20 Lakoff 1990: 6.

21 Lakoff 1990: 8.

22 Cf. Ortega y Gasset 1992: 115-16 y 1992: 226.

23 "Whenever a Dyirbal speaker uses a noun in a sentence, the noun must be preceded by a variant of one of four words: bayi, balan, balam, bala" (Lakoff 1990: 92). 
Dixon has provided a superb example of how human cognition works. Though the details of categorization may be unique to Dyirbal, the general principles at work in the Dyirbal system show up again and again in systems of human categorization ${ }^{24}$.

Y en la página siguiente corrobora:

Dixon's analysis explains why the Dyirbal system is the kind of system that human beings can function with.

Es decir, que según estas palabras, the kind of system (con el determinante artículo) quiere decir que no hay otro sistema "con el que los seres humanos puedan funcionar". En consecuencia, Lakoff considera la categorización del dyirbal como el tipo de categorización universal, prototípico y genuino.

2.8.6.2. Pero en esta interpretación de Lakoff tenemos un triple plano en el que Lakoff no repara. Una cosa es lo que la lengua dyirbal establece para su uso, lo cual deriva en una función gramatical dada, otra distinta es el significado que las palabras tienen en dyirbal, significado que necesariamente pertenece al universo de conocimiento dyirbal -por consiguiente, significado histórico-, y otra distinta, aquello que es motivo de connotación en la categoría gramatical referida. Ahora bien, ¿para quién o quiénes los objetos semánticos que forman la categoría balan constituyen motivo de connotación? La clase balan causa connotación precisamente en personas que no pertenecen al mundo dyirbal: en Lakoff y sus seguidores. Para éstos, el "fuego", el "agua" y la "lucha" constituyen cosas "peligrosas", por lo que "las mujeres", insinúan, aparecen también como cosas peligrosas. Pero este tipo de connotación es externo a la lengua dyirbal, puesto que la función de la clase balan es meramente gramatical, función que se manifiesta en el uso de la lengua. Al igual que en otras lenguas hay que marcar el género cuando se usan las palabras, que no los conceptos, (el maestro-la maestra), en dyirbal el hecho de que aparezcan las hembras junto al fuego y la lucha no encierra más valor que el de ser un requisito del uso, por tanto, una cosa gramatical convencional e histórica, que no tiene nada que ver con los objetos semánticos en los que se aplica ni con el significado que llevan consigo.

2.8.6.3. Clases de palabras semejantes a la clase balan del dyirbal se pueden encontrar, por ejemplo, en inglés. Cuando estudiamos la sintagmática ${ }^{25}$ de los adjetivos young, new y old, podremos ver que young y new, denotando el mismo significado léxico [edad: corta], se reparten en su combinación la oposición entre "lo vivo" (fundamentalmente lo humano) frente a lo "inerte", mientras que old, en sentido opuesto del significado léxico de los anteriores [edad: avanzada], se combina tanto con lo humano como con lo inerte. Es decir, young y new crean dos clases que se oponen entre sí separando "lo vivo (humano)" frente a "la cosa": $a$ young woman frente a new house. Pero old no hace esa distinción. Si hiciéramos consideraciones semejantes a las que hace Lakoff respecto a la clase balan del dyirbal, podríamos decir que old crea una realidad que no es lo más conveniente a lo que denota lo humano, puesto que si aparece junto con lo inerte algo tendrá en común, -aparte, claro está, del contenido semántico-, en virtud de lo cual llegan a constituir la misma clase. Y lo vivo y lo inerte se oponen polarmente entre sí: una cosa no puede ser a la vez viva e inerte. Podemos decir, pues, que el inglés tiene, por lo menos, una clase muy semejante a la del balan del

24 Lakoff 1990: 95.

25 La forma como se combinan las palabras entre sí dentro de un enunciado. 
dyirbal. Las palabras que pertenecen a ambas clases tienen su sintagmática aparte de su contenido semántico. El contenido semántico de las palabras, en el que se encuentra el clasema $^{26}$ que condiciona la sintagmática de las mismas, de las que componen la clase balan puede ser dispar entre sí -de hecho lo es-, como lo es el contenido semántico de 'young' y 'new' frente a 'old'.

2.8.6.4. Pero Lakoff explica esto que he llamado connotación basándose en el supuesto que él considera universalmente aceptado por quienes defienden la "teoría clásica de la categorías". Éste dice: "things are categorized together on the base of what they have in common". Como consecuencia de este principio añade:

The idea that categories are defined by common properties is not only our everyday folk theory of what a category is, it is also the principal technical theory -one that has been with us for more than two thousand years ${ }^{27}$.

Pero si el principio señalado arriba es falso, como defiende por todo su libro, no tiene ningún sentido buscar elementos que los miembros de la clase balan tienen en común. Es decir, que en la interpretación de este hecho de lengua Lakoff se apoya en el principio que niega. Justifica esta interpretación diciendo que "the chain of inference -from conjunction to categorization to commonality- is the norm". Aun siendo la norma, si el principio es falso no tiene sentido utilizarlo.

2.8.6.5. De cualquier forma Lakoff debería haber dicho quiénes son los que defienden el principio de que las categorías se forman por lo que las cosas tienen en común. Para Lakoff, el principio referido es un hecho que no explica, sino que meramente lo rechaza, atribuyéndolo a la teoría clásica de las teorías. Lakoff sugiere que las categorías de lo que llama la teoría clásica de las categorías están formadas por este principio que, insinúa igualmente, nos viene directamente de Aristóteles. Pero este principio ya en su propia formulación es contradictorio.

2.8.6.5.1. Lo primero que Lakoff debería haber hecho con este principio es analizar si existen categorías que se han formado según este principio. Lakoff en su estudio incluye incluso las categorías de las ciencias naturales, es decir, las "nomenclaturas" 28 , a las que considera como THE CATEGORIES OF THE WORLD, que añade como sugerencia, [they PSYCHOLOGICALLY] FIT THE CATEGORIES OF THE MIND ${ }^{29}$.

2.8.6.5.2. Pero tenemos que aclarar todo esto:

a) Las categorías de Aristóteles no tienen nada que ver con ese tipo de categorías de la teoría clásica. Las categorías de Aristóteles están estructuradas en torno al concepto de substancia, que es la primera de las categorías. Las otras categorías (el cuánto, el cuál, el relativo a, el dónde, el cuándo, el estar en una posición, el tener o estado, el hacer y el padecer), se han de entender en términos de la substancia, puesto que la forma de ser de éstas es ser en otra cosa (en otra substancia).

26 Cf. Coseriu 1981: 135 y 146-147; y Pottier 1976: 24.

27 Lakoff 1990: 5.

28 Coseriu llama a las clasificaciones objetivas, que no son lingüísticas sino científicas, "nomenclaturas", cf. Coseriu 1981: 95-107.

29 Lakoff 1990: 34. 
b) Cuando Lakoff habla del tipo de categorías de la teoría clásica formula la idea de que estas categorías han permanecido en la tradición durante el largo período de dos mil años. Además de en la cita de arriba, la idea aparece en distintas ocasiones en su libro ${ }^{30}$. Esto quiere decir, que, para Lakoff, las categorías de la teoría clásica son las que están en la tradición en la técnica del hablar, sin decirnos de qué técnica del hablar (=lengua) se trata. De hecho, en la tradición de una lengua hay muchas creencias, muchas categorías y muchos significados. Pero las categorías de la tradición ni son ni pueden ser de Aristóteles. Las categorías de la tradición son fundamentalmente los significados (de una lengua) y las que podemos llamar las categorías del conocer, conjunto de creencias comunes y participadas por los hablantes de una lengua. Si analizamos estas categorías veremos que en éstas no se aplica el principio de lo que las cosas tienen en común. Las categorías del conocer son necesarias para conocer y no se pueden formar por inducción (=método experimental=abstracción comunista, que dice Aristóteles), sino por creación. Las categorías del conocer están formadas de la misma manera como se forman los conceptos: por intuición y analogía.

Cuando conocemos por primera vez un objeto que no conocíamos, por ejemplo, un animal de otro continente, seleccionamos las características del mismo que en ese momento podemos juzgar esenciales y definitorias de forma intuitiva. Nos formamos así un concepto de lo que es ese animal y le atribuimos capacidad infinita de designación: todos los animales de su clase o especie serán de la misma forma (analogía). Ahora bien, cuando vemos un segundo ejemplar de esa clase comprobamos si ese nuevo ejemplar se acomoda o no a la definición inicial que nos habíamos formado. Si no se acomoda, corregimos entonces el concepto que inicialmente nos habíamos formado ${ }^{31}$. Por tanto en la formación del concepto o la categoría no hay posibilidad alguna de desarrollar un proceso inductivo (=método experimental) ni mucho menos de ver qué propiedades tienen en común las cosas que forman el concepto.

Por otro lado, este principio se revela en sí mismo imposible, porque antes de que exista la categoría no es posible aplicarlo ya que las cosas no existen como tales cosas más que cuando les aplicamos una categoría. La formación de una categoría, pues, es un acto de creación.

c) Las únicas categorías que han sido formadas o que son formadas por lo que las cosas tienen en común son aquellas de los lenguajes específicos (el lenguaje de los médicos, de los arquitectos, de los textos jurídicos, de la física, química, informática, etc., es decir, lenguajes específicos definidos por diferencias diastráticas). Pero estas categorías son científicas, no lingüísticas. Responden a clasificaciones objetivas de las cosas, en las que las cosas existían antes de formar la categoría científica. Con las categorías del conocer ocurre todo lo contrario: la cosa existe como tal cosa después de que sea creada la categoría y después de ser la cosa creada por la categoría. Es decir, tanto la categoría como las cosas se forman a la vez en el mismo acto del conocer. Es pues imposible que las categorías del conocer - y todas las categorías lingüísticas - se hayan formado por lo que las cosas tienen en común.

d) Por otro lado, las categorías que se transmiten en la tradición no pueden haber estado entre nosotros durante ese período de tiempo tan largo (dos mil trescientos años). Todo esto depende de lo que entendamos por lengua y estado de la lengua, conceptos que llevan consigo

30 Cf. Lakoff 1990: 5. La idea aparece también en la página 6: "From the time of Aristotle to the later work of Wittgenstein"; en la página 9: "These ideas have been part of the superstructure of Western intellectual life for two thousand years"; y en algunos otros lugares del libro.

31 Cf. Coseriu 1992: 226. En la página siguiente Coseriu dice: "El aprendizaje de la lengua por el niño, una permanente actividad creativa y un sacrificio: El niño sacrifica lo que ha creado él mismo cuando comprueba que no es eficaz en la comunicación con los adultos o con otros niños". 
el problema del cambio lingüístico, la sincronía y la evolución de una lengua. Las formas, procedimientos, reglas, contenidos y actitudes comunes y participadas de una lengua constituyen en sí mismos y en relación a los demás sistemas de isoglosas. El eje sincrónico en el hablar, distinto del eje de la evolución o diacrónico, mantiene una doble relación de solidaridad: de los hablantes entre sí y de los medios de expresión utilizados entre sí. La primera es la solidaridad con el interlocutor, solidaridad horizontal, y la segunda, solidaridad con la tradición, solidaridad vertical. La solidaridad horizontal da lugar a lo que llamamos el estado de la lengua, serie de sistemas de isoglosas que están en vigor en el hablar de una comunidad. La solidaridad vertical, para los hablantes de una lengua, significa permanencia de la tradición, por tanto, serie de reglas, procedimientos, contenidos, formas y actitudes comunes y participadas que los hablantes tienen como contenidos virtuales, según los cuales y en aproximación a los cuales crean a diario su hablar. Ahora bien, en el hablar de una comunidad de hablantes (=lengua) participan todos los hablantes, incluso aquellos que pertenecientes a otras lenguas hablan la lengua de la comunidad en la que están. Dada la naturaleza libre e histórica de los sujetos hablantes, el hablar nunca es igual: siempre es creativo, tanto en las formas materiales (palabras y contenidos) como en las formas propiamente virtuales (las reglas, procedimientos y actitudes participadas). La lengua como objeto histórico, objeto que manifiesta la historicidad de sus hablantes, cambia y se renueva en cada estado de la lengua. De esta manera una lengua histórica no es más que la sucesión de estados de la lengua que se reconstituyen ininterrumpidamente desde el estado de lengua anterior $^{32}$. Según esto no es cierto que las categorías hayan estado entre nosotros desde los tiempos de Aristóteles. Si alguna categoría queda, esa habrá evolucionado tanto que ya estará totalmente desfigurada. Pongamos un ejemplo. Infantil existe en las lenguas occidentales como palabra del presente estado de la lengua. Ahora bien, esta palabra tiene su origen en el indoeuropeo, ya que viene de la misma raíz que el verbo griego fari o femi, más el prefijo negativo in-. La combinación inicial era una metonimia, "el que no habla". Hoy día en algunas lenguas, por ejemplo en el español, ya no existe como substantivo ${ }^{33}$, pero sí existió así en el latín y hoy sigue existiendo en el inglés. El significado que hoy denota esa palabra ya no tiene nada que ver con hablar o no hablar, sino que es un significado nuevo, "relativo al niño o al bebé". Es decir, que como categoría ha desaparecido dando lugar a una nueva. Pero hay un hecho que es digno de mención en este caso: la palabra material ha sobrevivido a su contenido. Como categoría desapareció hace ya muchos años. Como palabra material es de plena vigencia.

2.8.6.5.3. Pero en el caso de las categorías de Aristóteles, los hechos contradicen ese aserto de la pervivencia de las categorías durante dos mil años. Cuando los comentaristas de Aristóteles (Porfirio, Ammonio Hermeiou, siglos III y VI después de Cristo) quisieron interpretar las categorías de Aristóteles ya no entendían la lengua de Aristóteles: habían pasado, cuando menos, unos seiscientos cincuenta años. Sólo cuando en el siglo XX Émile Benveniste nos dice que las categorías de Aristóteles no son lo que significan en su expresión literal, sino que hacen alusión a determinados recursos expresivos propios de la lengua de Aristóteles (el valor del verbo medio, la diátesis del perfecto, la correlación del adjetivo, etc.), es cuando las categorías de Aristóteles empiezan a tener sentido. De ninguna manera se puede defender que

Cf. Coseriu 1988.

Infante en español es hoy una palabra técnica, no de la lengua común. 
esas categorías u otras cualesquiera pueden haber pervivido durante tanto tiempo. Pero si alguna categoría ha pervivido este hecho hay que demostrarlo.

2.8.6.6. En el rechazo del principio de que las categorías se forman por lo que las cosas tienen en común por Lakoff tenemos cuatro cosas:

a) lo que es la categoría en sí (realidad universal de las categorías),

b) lo que es una categoría de una lengua (realidad histórica de las categorías),

c) lo que es una categoría del discurso (realidad individual, esporádica y contextual, de las categorías) y

d) lo que es el estudio de las categorías.

Lo que es la categoría en sí es una cosa absoluta: son instrumentos a priori del conocer; lo que es una categoría de una lengua es una realidad histórica, participada o contingente que va ligada a la significación y medios de expresión propios de la lengua; lo que es una categoría individual es una realidad contextual y esporádica; y lo que es el estudio de una categoría es un algo que pertenece a la teoría de las categorías. Las categorías históricas son por definición realidades contingentes y convencionales. Las únicas categorías formadas según el principio de lo que las cosas tienen en común son, como hemos dicho, las categorías que se dan o se pueden dar en los lenguajes específicos. Pero éstas no son lingüísticas, sino clasificaciones objetivas o "nomenclaturas". Volveremos sobre esto ${ }^{34}$.

2.9.1. Resumiendo lo dicho hasta ahora, las relaciones de significación que define Aristóteles en las Categorías son creaciones cognoscitivas fruto de un acto de conocer. En este sentido la realidad de las categorías es absoluta: son algo necesario para que se dé el acto del conocer, es decir, son instrumentos a priori del conocer. Y lo mismo podemos decir de la categoría balan y todas las categorías del dyirbal, que como categorías de una lengua son categorías históricas pero en sí mismas son absolutas. Y esto mismo vale para aquellas categorías que he llamado categorías del conocer: son realidades absolutas, algunas de las cuales se han hecho históricas pasando a la tradición. Las categorías que pueden estar formadas por el principio de lo que las cosas tienen en común son también absolutas cuando se emplean en el conocer, pero, al no ser lingüísticas, no son históricas ni pueden ser individuales. Éstas no responden a la sistematización de los hechos de la experiencia hecha por una lengua. Son distintas a todas las demás puesto que en su formación las cosas ya existían antes que la categoría: las cosas no necesitaban de la categoría para existir. En este sentido estas categorías no son delimitadoras de especies, no son lenguaje $\mathrm{e}^{35}$ sino nomenclaturas.

2.9.2. Lo dicho en este apartado nos suscita el siguiente problema: ¿Son las categorías categorías de la mente o categorías de las cosas?

Cf. $\S 3.1$ a 3.4 .

35 Dice Coseriu: “el lenguaje ciertamente no crea los entes, las 'cosas' naturales o de otra manera objetivas a las que atribuye un determinado modo de ser [...] [ni] el ser que se atribuye a las 'cosas': antes bien, reconoce y delimita modalidades del ser en las 'cosas' mismas y por eso es delimitación de especies o, [...] de 'clases' de entes (clases que, naturalmente, desde el punto de vista de su objetividad, pueden también ser clases de un solo miembro e incluso clases vacías)" (Coseriu 2006: 73-74). 


\section{Corolario}

\section{1. ¿Categorías de la mente o categorías de las cosas?}

Como corolario al problema de la realidad absoluta de las categorías, nos queda por ver si las categorías son categorías de la mente o, por el contrario, como defiende Lakoff ${ }^{36}$, son categorías de las cosas. Dicho sea de paso: decir categorías de la mente es redundante. Toda categoría es un concepto y todo concepto no puede ser más que mental. Es una fabricación de la mente.

Los cognitivistas dan por supuesto que las categorías son resultado de la categorización. Conciben la categorización como un mecanismo biológico que ha creado todas las categorías, con lo que lo absoluto de las categorías sería en sentido de natural ${ }^{37}$. Para los cognitivistas, la finalidad de las categorías es clasificar las cosas de la realidad, por lo que la base de la universalidad de las categorías son las cosas, siendo, por tanto, categorías de las cosas. Por otro lado, como hemos visto, atribuyen a Aristóteles lo que llaman la teoría clásica de las categorías $^{38}$. Ésta está constituida por las categorías formadas por lo que las cosas tienen en común. Según esto su propósito es corregir la concepción sobre las categorías que se basan en este criterio. Pero este tipo de teoría y el criterio en que se basa no responde a las categorías de Aristóteles. Sin embargo Lakoff insiste en que las categorías son categorías de las cosas. Lakoff quiere crear un nuevo concepto de categoría basado en las cosas tal y como son. Y en este intento tenemos una contradicción:

a) las categorías son abstractas por necesidad. Son elaboraciones mentales, es decir, conceptos y éstos no pueden ser más que abstractos;

b) las cosas no existen antes de las categorías. Una cosa es tal cuando a un "algo" se le aplica una abstracción creada (por analogía o creación pura), es decir, cuando se le aplica una categoría. Por consiguiente, esta pretensión de Lakoff de crear un concepto de categoría sobre la base de las cosas es una contradicción en sí misma. Las categorías son nocionales y las cosas se crean en el mismo acto en que se crean las categorías.

\subsection{Categorias del conocer.}

Puesto que Lakoff insiste en que las categorías de la teoría clásica "have been with us for more than two thousand years", podemos interpretar estas categorías como las categorías del conocer, creadas al hablar. Estas categorías se pueden poner de manifiesto, aunque no necesariamente ni todas, en el significado de una lengua. Las categorías del conocer, en contra de lo que dice Lakoff, no son categorías de Aristóteles ni tampoco están formadas por lo que las cosas tienen en común, ni llevan en la tradición tan largo período de tiempo. Podríamos referir estas categorías a Aristóteles si consideráramos los géneros y especies como categorías. En este caso podríamos ver una cierta relación entre las categorías de Aristóteles y

\section{Lakoff 1990: 9}

Cf. $§ 2.8 .1$ y ss.

38 En realidad, Lakoff nunca llega a decir "directamente" que la teoría clásica de las categorías es de Aristóteles; meramente lo deja entrever con expresiones como "the principal technical theory [...] that has been with us for more than two thousand years" (1990: 5), "from the time of Aristotle [...] categories were thought be well understood and unproblematic" (1990: 6), "These ideas have been part of the superstructure of Western intellectual life for two thousand years" (1990: 9), "They work by classical Aristotelian principles" (1990: 88), "linguists have simply taken for granted the classical theory of categorization, which has been with us since the time of Aristotle" (1990: 180). 
las categorías que refiere Lakoff que, para nosotros, son las categorías del conocer. Pero en este caso iríamos en contra la propia opinión de Aristóteles:

a. Aristóteles no considera a los géneros y especies como el tipo de categorías necesario para estudiar las cosas y hacer ciencia de ellas. Para Aristóteles, la substancia es la primera de las categorías, y él mismo la compara a la substancia segunda (los géneros y las especies). Dice que la verdadera es la substancia, no la substancia segunda. La substancia tiene un valor absoluto y admite contrarios ${ }^{39}$. Aristóteles no estaba interesado en los géneros y las especies, sino en individualizar las cosas para estudiarlas en sí mismas, haciendo con ello ciencia, cosa nueva frente a la filosofía imperante.

b. Los géneros y las especies, por otro lado, como categorías, están hechos por analogía, como hemos visto, lo mismo que las categorías del conocer.

c. Las categorías del conocer pueden pertenecer a la tradición, pero básicamente se crean en el mismo acto del conocer. Por otro lado, las que han pasado a la tradición no pueden haber durado tanto tiempo. Nunca una lengua ha durado en el mismo estado de lengua tanto tiempo.

Las categorías a las que se refiere Lakoff, que se conservan en la tradición, a las que he llamado las categorías del conocer, en cuanto tales categorías del conocer son absolutas. Sólo algunas de ellas han pasado a la tradición y hoy aparecen como históricas. Como realidades que se dan en el propio acto del conocer son creaciones esporádicas y efímeras que se dan de forma siempre nueva en el acto del conocer. Atribuir las categorías del conocer a Aristóteles equivale a decir que Aristóteles fue la primera persona sobre la faz de la tierra que llegó a conocer o que todos los humanos tras Aristóteles no han llegado aún a conocer.

\subsection{Lo que las cosas tienen en común y la experiencia.}

Veamos la crítica que hace Lakoff a las categorías de la teoría clásica, crítica que nos permite ver la atribución cognitivista de dicha teoría a Aristóteles:

From the time of Aristotle to the later work of Wittgenstein, categories were thought be well understood and unproblematic. They were assumed to be abstract containers, with things either inside or outside the category. Things were assumed to be in the same category if and only if they had certain properties in common. And the properties they had in common were taken as defining the category.

This classical theory was not the result of empirical study. It was not even a subject of major debate. It was a philosophical position arrived at on the basis of a priori speculation. Over the centuries it simply became part of the background assumptions taken for granted in most scholarly disciplines. In fact, until very recently, the classical theory of categories was not even thought of as a theory. It was taught in most disciplines not as an empirical hypothesis but as an unquestionable, definitional truth.

In a remarkably short time, all that has changed. Categorization has moved form the background to center stage because of empirical studies in a wide range of disciplines ${ }^{40}$.

3.3.1. Aquí nos interesa discutir únicamente si la base de los cognitivistas para referir las categorías de Aristóteles como categorías formadas por lo que las cosas tienen en común es 
cierta, por un lado, y si, por otro lado, lo que dice Lakoff se aplica de las categorías de Aristóteles.

3.3.2. Las categorías de Aristóteles son predicables, es decir, relaciones que son creadas por Aristóteles en el mero acto de decir de ellas con la pretensión de decir algo sobre el objeto al que se apliquen. Naturalmente las categorías de Aristóteles son abstractas: son relaciones cognoscitivas, es decir, conceptos, y como tales no pueden ser más que abstractas, al igual que los prototipos y categorías de los cognitivistas: éstos son también conceptos abstractos. Aristóteles, a pesar de dar por supuesto que las cosas son como se les considera que son, nunca trató de hacer una clasificación de las cosas, más bien, y como hemos visto, quiso hacer una propedéutica para estudiar las $\operatorname{cosas}^{41}$.

3.3.3. Los cognitivistas dicen que las categorías de Aristóteles no eran el resultado de la investigación empírica. Y ya hemos visto que el modo de pensar de Aristóteles estaba basado precisamente en la experiencia: fundaba el ser de las cosas en la acreditación de los sentidos. Para Aristóteles, las cosas son y son por la experiencia. Su modo de pensar era un modo sensual, es decir, Aristóteles tenía que asegurarse en los sentidos para dar algo por verdadero. Y el método experimental, del cual hacen bandera los cognitivistas en contra de Aristóteles, no era tan extraño a Aristóteles como los cognitivistas presumen. Ortega y Gasset destaca la "abstracción comunista" como la forma de proceder de Aristóteles en su búsqueda del Saber $^{42}$. A pesar de esto las categorías de Aristóteles no eran categorías de las cosas, porque Aristóteles no utilizó la inducción, es decir, el partir de los individuales para su definición, sino por reflexión, basándose en el saber originario que como hablante tenía sobre su propia lengua.

\subsection{Categorías abstractas y conceptos físicos.}

3.4.1. Por otro lado, postular, como Lakoff, que tenemos categorías tanto concretas como abstractas: "Any adequate account of human thought must provide an accurate theory for all our categories, both concrete and abstract" ${ }^{\prime 43}$, es una cosa mucho más sorprendente que el famoso título del libro de Lakoff. ¿Puede haber categorías concretas? Lo concreto y lo abstracto son términos incompatibles entre sí, términos absolutos en el que el uno excluye al otro. Aceptar que hay categorías concretas y categorías abstractas, quiere decir que en el concepto de categoría se da a la vez lo abstracto y lo concreto, con lo que es contradictorio consigo mismo. El conocer es una actividad mental que parte de lo concreto y lo transforma en algo que no es ni existe con existencia concreta.

3.4.2. Por otro lado, las substancias segundas, es decir, los géneros y las especies, tienen una designación. Los géneros y las especies son consideraciones cognoscitivas, es decir, conceptos, con designación en las cosas reales. El contenido de un concepto es una cosa y la designación de mismo, otra muy distinta. Así, podremos hablar del hombre, y podremos buscar el concepto hombre en los individuales. Pero esto no será una categoría formal de Aristóteles, será un acto mental elaborado por alguien que signifique, por un lado, y que designe, por otro. La designación sí, trata de las cosas reales, en donde se dan las cosas concretas. Lo que tenemos que hacer es no confundir planos. Pero lo que en Aristóteles no se

$41 \quad$ Cf. $\S 4.2$.

42 Ortega y Gasset 1992: 115-16.

43 Lakoff 1990: 6 
aplica de ninguna manera es que haya conceptos físicos: "It is obvious that not all of our concepts are physical concepts. Many of them are abstract. Reason is abstract ${ }^{44, "}$.

\section{Las categorías, creación cognoscitiva.}

Pero tenemos que buscar la realidad de las categorías allá en donde se dé. Cuando hablamos, hablamos utilizando las categorías que nos inventamos en el mismo acto del hablar, que es un acto de hablar, decir y conocer. En este sentido cada hablante, en cada acto del habla, crea una categoría nueva. Pretender ver una identidad entre lo concebido y lo dicho con las cosas del mundo real, no tiene sentido. El lenguaje es la creación de significados que emplea signos. Lo creado es mental, es decir, abstracto por naturaleza. En su realidad universal y absoluta las categorías son instrumentos a priori del conocer, es decir, instrumentos necesarios del conocer, sin los cuales no se daría el conocer y, al contrario: no se darían las categorías si no existiera el conocer (humano), pues el conocer consiste precisamente en eso: en crear y separar, es decir, en crear categorías para separar aspectos que creamos sobre lo que percibimos, lo que nos rodea, a lo cual llamamos la realidad.

\section{Las categorías y el pensamiento.}

5.1. Aristóteles no llegó a plantearse el problema de las relaciones lenguaje-pensamiento. Este problema no tenía cabida dentro del modo de pensar de Aristóteles pues Aristóteles identificaba la realidad con lo conocido ${ }^{45}$. No obstante, Aristóteles no es ajeno a separar lo que da el lenguaje en contraposición a aquello para lo que sirve el lenguaje: los distintos usos. En De interpretatione defiende la función significativa del lenguaje frente al uso del mismo. Para Aristóteles, no existe la realidad como distinta del pensamiento, con lo que el lenguaje necesariamente tenía que acomodarse a esa doble realidad. La lengua, para Aristóteles, es una cosa dada sin posibilidad de que no reflejara la mente. Pero cuando Aristóteles hace la distinción entre lenguaje como significativo y el lenguaje como uso, habla del lenguaje ulteriormente determinado, lo cual nos dice lo siguiente: el lenguaje es una realidad determinable, es decir, una actividad libre.

5.2. Para Aristóteles, en consecuencia, el lenguaje es un fiel reflejo de la mente y de la realidad. No obstante, en De interpretatione Aristóteles define el lenguaje como lo meramente significativo separándolo de lo lógico. El procedimiento de esta separación está en la combinación o no de las palabras entre sí. Esta concatenación de realidades (casi identidad de la mente y la realidad y casi identidad del lenguaje y la mente) lleva a Aristóteles a fijarse en aquellas matizaciones o relaciones que constituyen como previas el estudio de las cosas tal y como se dan en sí mismas. Las relaciones que encuentra, las categorías, son relaciones de significación que están en todas las cosas. La razón de este estar en todas las cosas está en lo que Aristóteles es capaz de distinguir según los parámetros de la lengua griega, sin que Aristóteles se diera cuenta de que tales relaciones de significación eran propias de la lengua griega y no de otra lengua. Esto lleva consigo una identificación entre la mente que es capaz de identificar esa relación significativa y la lengua. Para Aristóteles, lo hablado se identifica con lo pensado y lo pensado con lo real: describir esas relaciones significativas era descubrir

$44 \quad$ Lakoff 1990: 267.

45 Cf. Ortega y Gasset 1992: 29. 
lo que se daba en el pensamiento y por tanto en las cosas. El estudio individual de las cosas comenzaba con el descubrimiento de la substancia, el cuánto, el cuál, el dónde, etc.

\section{El ser griego y la substancia de Aristóteles.}

6.1. Por otro lado, Aristóteles acepta la existencia de las cosas. Éstas son algo objetivo y concreto, la viva representación de la substancia. La prueba de la existencia de las cosas era la acreditación de los sentidos ${ }^{46}$. Percibimos las cosas sensorialmente. Por otro lado, la gente acepta igualmente las cosas en sí mismas. Por tanto, son. Aristóteles concibe lo que es doblemente: como ente en sí mismo (algo que es) y como algo objetivo (lo que es es cosa). La base de esta concepción es el concepto de ser de la tradición griega, el ser substante. Para Párménides, el ser es uno, eterno, inmóvil, indiviso, no tiene principio ni fin ${ }^{47}$. Aristóteles busca el ser en las cosas y les atribuye a cada una el concepto de ser como condición de su existencia, creando así el ser como substancia: "el verdadero o más auténtico Ente es la substancia, y la substancia es 'este hombre', 'este caballo' que veo. Nada más”"48. Aristóteles añade al ser de Parménides el que sea por sí mismo y no necesite de otro ser para ser, es decir, que Aristóteles transforma el ser de Parménides en substancia. De aquí, que el primer predicable sea el que una cosa sea por sí misma y como cosa objetiva, es decir, que la cosa sea una substancia. Las cosas, que para Aristóteles están ahí, lo primero que necesitan no es su esencia (categoría) sino su individuación como entes, es decir, como substancia. A partir de la concepción de las cosas como substancia, la cosa entra en el campo del Saber y podemos ver en ella los otros predicables. Para Aristóteles, el ser es "el primum cogitabile"49. Pero el ser, para Aristóteles, es algo que está ejecutando su propia esencia, algo que es siendo: es el ser en acto, Ł̇vé $\rho \gamma \in \epsilon^{\prime} \alpha$ ỏv. Ortega resume el concepto de ser de Aristóteles frente al concepto de ser de la tradición griega de la siguiente manera:

La concepción griega del ser [lo que hay tras lo que hay, lo que tras-hay] posee, ciertamente un lado estático que le viene no tanto de que se orienta en los objetos según están ante él y le son meros aspectos o espectáculos, sino a causa de la fijación "cristalización" que en ellos pone el concepto. El concepto es inmóvil (idéntico a sí mismo); no varía, no se esfuerza, no vive. Es lo que ya es, y nada más. Pero el Ser en los griegos, aun poseyendo esa fijeza y parálisis que del concepto le sobreviene cuya proyección en el plano de "la existencia fuera" (Tó éKTós, tó ektós) es- consiste en, permítaseme la expresión, en estar haciendo su esencia, en estarla ejecutando. Este lado del ser -frente al del estatismo- aparece oficialmente formulado en la idea aristotélica del Ser como actualidad:

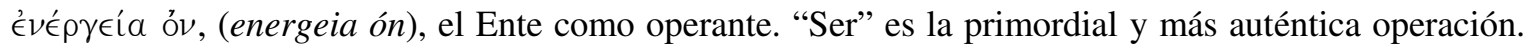
"Ser caballo" no es sólo presentar al hombre la forma visible "caballo", sino estarla siendo desde dentro, estar haciendo o sosteniendo en el ámbito ontológico su "cabalidad"; en suma, ser caballo es "caballear", como ser flor es "florear" y ser color, "colorear". El Ser en Aristóteles tiene valencia de verbo activo ${ }^{50}$.

El ser de Aristóteles es el ser en el que el sujeto que lo concibe implica la realidad. El

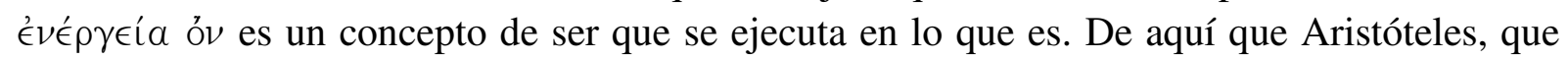

46 Ortega y Gasset 1992: 254.

47 Cf. Ferrater Mora, Diccionario de filosofía.

48 Ortega y Gasset 1992: 239.

49 Ortega y Gasset 1992: 216.

50 Ortega y Gasset 1992: 277-78. 
era más científico que filósofo ${ }^{51}$, intuyera la necesidad de estudiar las cosas una por una, es decir, individualmente, frente a las Ideas de su maestro Platón. Con el esquema de relaciones descritas en las Categorías Aristóteles abría la puerta a lo que todavía no se había hecho: la ciencia, algo nuevo frente a la filosofía.

6.2. Esta concepción sobre el ser que es siendo lo que es invita a Aristóteles a analizar todo cuanto es predicable del ser. Las cosas habían pasado de estar estáticamente en el mundo a presentarse ante el observador. Para Aristóteles, la substancia es un algo que está ahí y se presenta ante nosotros, porque es siendo. Este algo objetivo y dinámico a la vez, es un algo que es preciso definir antes de hacer una ciencia particular. Antes que considerar si un caballo "caballea" es necesario poner de manifiesto todas las relaciones que se predican de él en cuanto ser que es en sí (substancia). Los demás predicables tienen que ver con la substancia, precisamente, por el modo de ser de los mismos. Pero todos los predicables participan del ser substancia de un modo u otro. El cuánto y el cuál es un algo que necesita apoyarse en la substancia para ser. Los otros predicables también son ser, pero de un modo distinto: el relativo a establece una relación entre dos substancias pero de tal forma que la relación está ahí y se nos presenta ante nosotros. El dónde y el cuándo definen la relación del ente con el espacio y el tiempo porque el problema fundamental del Ente es su existir. No se da el Ente sino los entes. El Ente así es el Existente. Las cosas se diferencian entre sí no sólo por lo que son (por pertenecer a una especie, que las diferencia de las demás de cosas de otras especies, es decir, por ser definidas por una categoría), sino también por el modo de ser eso que son, con lo que las cosas, además de su esencia, tienen existencia individual, con lo que se diferencian entre sí como seres individuales ${ }^{52}$. Y este descubrimiento, el Ser en cuanto el Existente, es, para Ortega, una de las tres máximas "averiguaciones" de la filosofía griega. Dice Ortega al respecto:

Con ejemplar agudeza [Aristóteles] había descubierto que si repartimos las cosas todas en grandes clases, hallaremos que aquellas no se diferencian entre sí sólo por lo que son -por tanto, como una especie de otra-, sino también por el modo de ser eso que son. La blancura no sólo se diferencia del caballo en que aquella es un color y éste un animal, etcétera, sino en que el existir de la blancura es apoyarse en otra cosa sin la cual no puede ser, mientras que para el caballo existir es estar atenido a sí propio y no en apoyarse en otra cosa. Esta advertencia perspicacísima hubiera sido imposible si antes no hubiera hecho su máximo descubrimiento, que él solo compensa sus otras deficiencias, y que con el descubrimiento de Ente en Parménides y el descubrimiento del lógos o Razón en Platón, constituyen las tres máximas averiguaciones que debemos a la filosofía griega. Me refiero al descubrimiento de que el problema radical del Ente es su existir, o, dicho en la terminología moderna [...]: que el problema radical de lo Real es su realidad ${ }^{53}$.

6.3. Categorías tales como el dónde y el cuál no están relacionadas con el Ente, sino con el Existente, es decir, no hablan de la realidad de la substancia de la que se predican. Tampoco

51 “Aristóteles era un hombre de ciencia, y fue filósofo en tanto que hombre de ciencia. Su reforma del platonismo consistió en declarar urgente el conocimiento de las cosas concretas que 'están ahí' y nos rodean por todas partes" (Ortega y Gasset 1992: 126).

52 No obstante, Aristóteles nos dirá que la relación del Ente con las cosas en donde se da es analógica, con lo que crea un pseudogénero (el género que incluiría al ser en cuanto ser, en donde ningún ente se diferenciaría de los demás). Cf. Ortega y Gasset 1992: 219-221.

53 1992: 220-21. 
el dónde y el cuándo hablan de la substancia sino del Existente, por tanto refieren algo que es susceptible de definición individual.

\section{Simetría de las categorías.}

\subsection{Simetría de las categorías de Aristóteles.}

Una última cuestión sobre la realidad de las categorías. ¿Son las categorías de Aristóteles intercambiables entre sí? ¿Son las categorías de Aristóteles simétricas? Las categorías de Aristóteles no son simétricas, es decir, no son intercambiables entre sí: designan realidades que sólo se pueden explicar en términos de lo que es la primera de ellas, la substancia. Dicho de otro modo: Aristóteles emplea un doble criterio para la creación de las categorías: el modo de ser en sí (la substancia) y el modo de ser en otra cosa (las restantes categorías).

\subsection{Simetría de las categorías de los cognitivistas.}

La misma cuestión tenemos que hacérnosla respectos a las categorías de Lakoff. Éstas en mucho mayor grado no son simétricas. En cuanto a la formación de las categorías por Lakoff, éste, al igual que Aristóteles concibiera en su Saber, sigue el mismo método: partir de principios que no se discuten. Así, Lakoff crea lo que es su teoría sobre las categorías sobre la base de las objeciones que él hace a las que considera las "categorías de la teoría clásica". Estas objeciones son principios sobre los que fundamenta su "nuevo" concepto de categoría, a saber: "family resemblances", “centrality”, "polysemy as categorization”, "generality as a prototype phenomenon", "membership gradience", "centrality gradience”, "conceptual embodiment", "functional embodiment", "basic-level categorization", "basic-level primacy", "reference-point, or 'metonymic', reasoning”. El criterio de formación de las categorías de Lakoff es múltiple y sin cohesión entre los distintos elementos que lo componen.

\section{Bibliografía utilizada}

Aristóteles (2009): Categorías, Sobre la interpretación. Introducción, traducción y notas: Jorge Mittelmann. Editorial Losada.

- (2008): Metafísica. Alianza Editorial: Clásicos de Grecia y Roma.

Coseriu, Eugenio (1981) [1977]: Principios de semántica estructural. Madrid: Editorial Gredos.

- (1988) [1957]: Sincronía, diacronía e historia: el problema del cambio lingüístico. Madrid: Editorial Gredos.

- (1992) [1988]: Competencia lingüística: elementos de la teoría del hablar. Madrid: Editorial Gredos.

Coseriu, E./ Ó. Loureda (2006): Lenguaje y discurso. Pamplona: Eunsa.

Ferrater Mora, José: Diccionario de filosofía, 5 tomos. RBA.

Humboldt, W. (1990 [1836]): Ueber die Verschiedenheit des menschlichen Sprachbaues und ihren Einfluss auf die geistige Entwickelung des Menschengesschlechts, traducción española de Ana Agud: Sobre la diversidad de la estructura del lenguaje humano y su influencia sobre el desarrollo espiritual de la humanidad. Madrid: Anthropos y Ministerio de Educación y Ciencia. 
Martínez del Castillo, J. G. (1999): La intelección, el significado, los adjetivos. Almería: Servicio de Publicaciones de la Universidad de Almería.

- (2004): La lingüística del decir: el logos semántico y el logos apofántico. Granada,

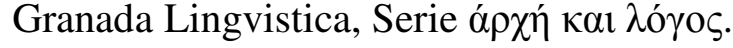

- (2008): La lingüística cognitiva: análisis y revisión. Madrid, Biblioteca Nueva, S. A.

Mittelmann, Jorge (2009): "Introducción” en Aristóteles (2009).

Ortega y Gasset, José (1989) [1981]: Origen y epílogo de la filosofía. Revista de Occidente en Alianza Editorial.

- (1992) [1958]: La idea de principio en Leibniz. Revista de Occidente en Alianza Editorial.

- (1996) [1979]: Sobre la razón histórica. Revista de Occidente en Alianza Editorial.

Popper, Karl (2002): The Logic of Scientific Discovery. Londres: Taylor \& Francis Ltd.

Pottier, Bernard. (1976) [1974]. Lingüística general: teoría y descripción. Madrid: Editorial Gredos. 